\title{
Inequalities for the Polar Derivative of a Polynomial
}

\author{
Gulshan Singh ${ }^{1}$, Wali Mohammad Shah ${ }^{2}$, Yash Paul ${ }^{1}$ \\ ${ }^{1}$ Bharathiar University Coimbatore, Tamil Nadu, India \\ ${ }^{2}$ Department of Mathematics, Kashmir University, Srinagar, India \\ E-mail: \{gulshansingh1,wmshah, yashpaul2011\}@rediffmail.com \\ Received February 20, 2011; revised March 1, 2011; accepted March 5, 2011
}

\section{Abstract}

If $P(z):=\sum_{j=0}^{n} a_{j} z^{j}$ is a polynomial of degree $n$, having all its zeros in $|z| \leq K, K \geq 1$, then it was provied by Aziz and Rather [2] that for every real or complex number $\alpha$ with $|\alpha| \geq K, \operatorname{Max}_{|z|=1}\left|D_{\alpha} P(z)\right| \geq$ $\frac{n(|\alpha|-K)}{\left(K^{n}+1\right)} \operatorname{Max}_{|z|=1}|P(z)|$. In this paper, we sharpen above result for the polynomials $P(z)$ of degree $n>3$.

Keywords: Polynomial, Inequality, Polar Derivative

\section{Introduction}

Let $P(z):=\sum_{j=0}^{n} a_{j} z^{j}$ be a polynomial of degree $\mathrm{n}$ and $P^{\prime}(z)$ its derivative, then

$$
\operatorname{Max}_{|z|=1}\left|P^{\prime}(z)\right| \leq \operatorname{Max}_{|z|=1}|P(z)|
$$

Inequality (1) is a famous result due to Bernstein and is best possible with equality holding for the polynomial $P(z)=\lambda z^{n}$, where $\lambda$ is a complex number.

If we restricted ourselves to a class of polynomial having no zeros in $|z|<1$, then the above inequality can be sharpened. In fact, Erdös conjectured and later Lax [6] proved that if $P(z) \neq 0$ in $|z|<1$, then

$$
\operatorname{Max}_{|z|=1}\left|P^{\prime}(z)\right| \leq \frac{n}{2} \operatorname{Max}_{|z|=1}|P(z)|
$$

On the other hand, it was proved by Turán [10] that if $P(z)$ is a polynomial of degree $\mathrm{n}$ having all its zeros in $|z| \leq 1$, then

$$
\operatorname{Max}_{|z|=1}\left|P^{\prime}(z)\right| \geq \frac{n}{2} \operatorname{Max}_{|z|=1}|P(z)|
$$

The inequalities (2) and (3) are also best possible and become equality for polynomials which have all zeros on $|z|=1$.

For the class of polynomials having all the zeros in $|z| \leq K$, Malik [7] (See also Govil [5]) proved that if $P(z)$ is a polynomial of degree $\mathrm{n}$ having all zeros lie in $|z| \leq K$, then

$$
\operatorname{Max}_{|z|=1}\left|P^{\prime}(z)\right| \geq \frac{n}{1+K} \operatorname{Max}_{|z|=1}|P(z)| \text {, if } K \leq 1,
$$

where as Govil [5] showed that

$$
\operatorname{Max}_{|z|=1}\left|P^{\prime}(z)\right| \geq \frac{n}{1+K^{n}} \operatorname{Max}_{|z|=1}|P(z)| \text {, if } K \geq 1
$$

Both the inequalities are best possible, with equality in (4) holding for $P(z)=(z+K)^{n}$ and in (5) the equality holds for the polynomial $P(z)=\left(z^{n}+K^{n}\right)$.

Let $D_{\alpha} P(z)$ denote the polar derivative of the polynomial $P(z)$ of degree $\mathrm{n}$ with respect to $\alpha$, then

$$
D_{\alpha} P(z)=n P(z)+(\alpha-z) P^{\prime}(z) .
$$

The polynomial $D_{\alpha} P(z)$ is of degree at most $n-1$ and it generalizes the ordinary derivative in the sense that

$$
\lim _{\alpha \rightarrow \infty} \frac{D_{\alpha} P(z)}{\alpha}=P^{\prime}(z) .
$$

Aziz and Rather [2] extended (5) to the polar derivative of a polynomial and proved the following:

Theorem 1: If the polynomial $P(z):=\sum_{j=0}^{n} a_{j} z^{j}$ has all its zeros in $|z| \leq K, K \geq 1$, then for every real or complex number $\alpha$ with $|\alpha| \geq K$,

$$
\operatorname{Max}_{|z|=1}\left|D_{\alpha} P(z)\right| \geq \frac{n(|\alpha|-K)}{\left(K^{n}+1\right)} \operatorname{Max}_{|z|=1}|P(z)|
$$

In this paper, we prove the following result which is a refinement as well as generalization of Theorem 1 . 
Theorem 2: Let $P(z):=\sum_{j=0}^{n} a_{j} z^{j}, a_{n} a_{0} \neq 0$ be a polynomial of degree $n>3$, having all its zeros in $|z| \leq K$,

$K \geq 1$, then for every real or complex number $\alpha$ with $|\alpha| \geq K$

$$
\begin{aligned}
\operatorname{Max}_{|z|=1}\left|D_{\alpha} P(z)\right| \geq & \frac{n(|\alpha|-K)}{\left(K^{n}+1\right)}\left\{\operatorname{Max}_{|z|=1}|P(z)|+\operatorname{Min}_{|z|=K}|P(z)|+\frac{2\left|a_{n-1}\right|}{(n+1)} \mid \frac{\left(K^{n}-1\right)}{n}-(K-1)\right] \\
& +2\left|a_{n-2}\right|\left[\left\{\frac{\left(K^{n}-1\right)-n(K-1)}{n(n-1)}\right\}-\left\{\frac{\left(K^{n-2}-1\right)-(n-2)(K-1)}{(n-2)(n-3)}\right\}\right], \text { if } n>3 .
\end{aligned}
$$

Remark 1: For $K=1$, Theorem 2 provides a refiment of a theorem proved by Shah [9].

Remark 2: For $K>1$, and for $y>1$,

$\frac{\left[\left(K^{y}-1\right)-y(K-1)\right]}{y(y-1)}$ and $\frac{\left(K^{y}-1\right)}{y}$ are both increasing functions of $y$ and so the expressions

$\left[\left\{\frac{\left(K^{n}-1\right)-n(K-1)}{n(n-1)}\right\}-\left\{\frac{\left(K^{n-2}-1\right)-(n-2)(K-1)}{(n-2)(n-3)}\right\}\right]$

$$
\left[\frac{\left(K^{n}-1\right)}{n}-(K-1)\right]
$$

and

are always non-negative so that for polynomials of degree $n>3$, Theorem 2 is an improvement of Theorem 1.

Dividing both sides of (7) by $|\alpha|$ and letting $|\alpha| \rightarrow \infty$, we get the following:

Corollary 1: Let $P(z)=\sum_{j=0}^{n} a_{j} z^{j}, a_{n} a_{0} \neq 0$ be a polynomial of degree $n>3$, having all its zeros in $|z| \leq K$, $K \geq 1$, then

$$
\begin{aligned}
\operatorname{Max}_{|z|=1}\left|P^{\prime}(z)\right| \geq & \frac{n}{\left(K^{n}+1\right)}\left\{\operatorname{Max}_{|z|=1}|P(z)|+\operatorname{Min}_{|z|=K}|P(z)|+\frac{2\left|a_{n-1}\right|}{(n+1)} \mid \frac{\left(K^{n}-1\right)}{n}-(K-1)\right] \\
& \left.+2\left|a_{n-2}\right|\left[\left\{\frac{\left(K^{n}-1\right)-n(K-1)}{n(n-1)}\right\}-\left\{\frac{\left(K^{n-2}-1\right)-(n-2)(K-1)}{(n-2)(n-3)}\right\}\right]\right\}, \text { if } n>3 .
\end{aligned}
$$

\section{Lemmas}

We need the following lemmas.

Lemma 1: Let $P(z)$ be a polynomial of degree $n$, then for $R \geq 1$.

$$
\operatorname{Max}_{|z|=R}|P(z)| \leq R^{n} \operatorname{Max}_{|z|=1}|P(z)| .
$$

The above lemma is a simple consequence of the maximum modulus principle [8].
Lemma 2: If $P(z):=\sum_{j=0}^{n} a_{j} z^{j}, a_{n} \neq 0$, is a polynomial of degree $n$ having all its zeros in $|z| \leq 1$, then

$$
\operatorname{Max}_{|z|=1}\left|P^{\prime}(z)\right| \geq \frac{n}{2}\left\{\operatorname{Max}_{|z|=1}|P(z)|+\operatorname{Min}_{|z|=1}|P(z)|\right\} .
$$

This lemma is due to Aziz and Dawood [1].

Lemma 3: If $P(z):=\sum_{j=0}^{n} a_{j} z^{j}$ is a polynomial of degree $n$ having no zeros in $|z| \leq 1$, and $m=\operatorname{Min}_{|z|=1}|P(z)|$, then for $R \geq 1$ and $n>3$,

$$
\begin{aligned}
M(P, R) \leq & \frac{\left(R^{n}+1\right)}{2} \operatorname{Max}_{|z|=1}|P(z)|-\frac{\left(R^{n}-1\right)}{2} m-\frac{2\left|P^{\prime}(0)\right|}{(n+1)}\left[\frac{\left(R^{n}-1\right)}{n}-(R-1)\right] \\
& \left.-\left|P^{\prime \prime}(0)\right|\left\{\frac{\left(R^{n}-1\right)-n(R-1)}{n(n-1)}\right\}-\left\{\frac{\left(R^{n-2}-1\right)-(n-2)(R-1)}{(n-2)(n-3)}\right\}\right]
\end{aligned}
$$

The above result is a special case of a result due to Dewan, Singh and Mir [4, Theorem 1] with $K=1$ and $\mu=1$.
Remark 3: Here we note that for the proof of this result an additional hypothesis that $P(0) \neq 0$ is required. A simple counter example in this case is $P(z)=z^{n}$. 


\section{Proof of Theorem 2}

Since $P(z)$ has all its zeros in $|z| \leq K$, therefore $G(z)=P(K z)$ has all its zeros in $|z| \leq 1$ and hence by applying lemma 2 to the polynomial $G(z)$, we get

$$
\operatorname{Max}_{|z|=1}\left|G^{\prime}(z)\right| \geq \frac{n}{2}\left\{\operatorname{Max}_{|z|=1}|G(z)|+\operatorname{Min}_{|z|=1}|G(z)|\right\} \text {. }
$$

Let $H(z)=z^{n} G\left(\frac{1}{\bar{z}}\right)$. Then it can be easily verified that

$$
\left|H^{\prime}(z)\right|=\left|n G(z)-z G^{\prime}(z)\right|, \text { for }|z|=1 .
$$

The polynomial $H(z)$ has all its zeros in $|z| \geq 1$ and $|H(z)|=|G(z)|$ for $|z|=1$, therefore, by result of a de Bruijn [3]

$$
\left|H^{\prime}(z)\right| \leq\left|G^{\prime}(z)\right| \text { for }|z|=1
$$

Now for every real or complex number $\alpha$ with $|\alpha| \geq K$, we have

$$
\begin{aligned}
\left|D_{\frac{\alpha}{K}} G(z)\right| & =\left|n G(z)-z G^{\prime}(z)+\frac{\alpha}{K} G^{\prime}(z)\right| \\
& \geq\left|\frac{\alpha}{K}\right|\left|G^{\prime}(z)\right|-\left|n G(z)-z G^{\prime}(z)\right|
\end{aligned}
$$

For this, we get by using (10) and (11)

$$
\operatorname{Max}_{|z|=1}\left|D_{\frac{\alpha}{K}} G(z)\right| \geq \frac{|\alpha|-K}{K} \operatorname{Max}_{|z|=1}\left|G^{\prime}(z)\right|
$$

Using (9) in (12), we get

$$
\operatorname{Max}_{|z|=1}\left|D_{\frac{\alpha}{K}} G(z)\right| \geq \frac{(|\alpha|-K)}{K} \frac{n}{2}\left\{\operatorname{Max}_{|z|=1}|G(z)|+\operatorname{Min}_{|z|=1}|G(z)|\right\} .
$$

Replacing $G(z)$ by $P(K z)$, we have

This gives

$$
\operatorname{Max}_{|z|=1}\left|D_{\frac{\alpha}{K}} P(K z)\right| \geq \frac{n(|\alpha|-K)}{2 K}\left\{\operatorname{Max}_{|z|=1}|P(K z)|+\operatorname{Min}_{|z|=1}|P(K z)|\right\} .
$$

$$
\operatorname{Max}_{|z|=1}\left|n P(K z)+\left(\frac{\alpha}{K}-z\right) K P^{\prime}(K z)\right| \geq \frac{n(|\alpha|-K)}{2 K}\left\{\operatorname{Max}_{|z|=1}|P(K z)|+\operatorname{Min}_{|z|=1}|P(K z)|\right\} .
$$

Equivalently

$$
\operatorname{Max}_{|z|=K}\left|D_{\alpha} P(z)\right| \geq \frac{n(|\alpha|-K)}{2 K}\left\{\operatorname{Max}_{|z|=K}|P(z)|+\operatorname{Min}_{|z|=K}|P(z)|\right\} .
$$

Since the polynomial $\mathrm{P}(\mathrm{z})$ has all its zeros in $|z| \leq K, K \geq 1$. If $Q(z)=z^{n} P\left(\frac{1}{z}\right)$ be the reciprocal polynomial of $P(z)$. Then the polynomial $Q\left(\frac{z}{K}\right)$ has

$$
\begin{aligned}
\operatorname{Max}_{|z|=K}\left|Q\left(\frac{z}{K}\right)\right| \leq & \left.\frac{\left(K^{n}+1\right)}{2} \operatorname{Max}_{|z|=1}\left|Q\left(\frac{z}{K}\right)\right|-\frac{\left(K^{n}-1\right)}{2} \operatorname{Min}_{|z|=1}\left|Q\left(\frac{z}{K}\right)\right|-\frac{2\left|a_{n-1}\right|}{(n+1)} \mid \frac{\left(K^{n}-1\right)}{n}-(K-1)\right] \\
& -2\left|a_{n-2}\right|\left[\left\{\frac{\left(K^{n}-1\right)-n(K-1)}{n(n-1)}\right\}-\left\{\frac{\left(K^{n-2}-1\right)-(n-2)(K-1)}{(n-2)(n-3)}\right\}\right]
\end{aligned}
$$

all its zeros in $|z| \geq 1$. Hence applying lemma 3 to the polynomial $Q\left(\frac{z}{K}\right), K \geq 1$, we get

This in particular gives

$$
\begin{aligned}
\operatorname{Max}_{|z|=1}|P(z)| \leq & \left.\frac{\left(K^{n}+1\right)}{2 K^{n}} \operatorname{Max}_{|z|=K}|P(z)|-\frac{\left(K^{n}-1\right)}{2 K^{n}} \operatorname{Min}_{|z|=K}|P(z)|-\frac{2\left|a_{n-1}\right|}{(n+1)} \mid \frac{\left(K^{n}-1\right)}{n}-(K-1)\right] \\
& -2\left|a_{n-2}\right|\left[\left\{\frac{\left(K^{n}-1\right)-n(K-1)}{n(n-1)}\right\}-\left\{\frac{\left(K^{n-2}-1\right)-(n-2)(K-1)}{(n-2)(n-3)}\right\}\right]
\end{aligned}
$$


which is equivalent to

$$
\begin{aligned}
\operatorname{Max}_{|z|=K}|P(z)| \geq & \frac{2 K^{n}}{\left(K^{n}+1\right)} \operatorname{Max}_{|z|=1}|P(z)|+\frac{\left(K^{n}-1\right)}{\left(K^{n}+1\right)} \operatorname{Min}_{|z|=K}|P(z)|+\frac{4 K^{n}}{\left(K^{n}+1\right)} \frac{\left|a_{n-1}\right|}{(n+1)}\left[\frac{\left(K^{n}-1\right)}{n}-(K-1)\right] \\
& +\frac{4 K^{n}}{\left(K^{n}+1\right)}\left|a_{n-2}\right|\left[\left\{\frac{\left(K^{n}-1\right)-n(K-1)}{n(n-1)}\right\}-\left\{\frac{\left(K^{n-2}-1\right)-(n-2)(K-1)}{(n-2)(n-3)}\right\}\right]
\end{aligned}
$$

Using (14) in (13), we get

$$
\begin{aligned}
\operatorname{Max}_{|z|=K}\left|D_{\alpha} P(z)\right| \geq & \frac{n(|\alpha|-K)}{2 K}\left\{\frac{2 K^{n}}{\left(K^{n}+1\right)} \operatorname{Max}_{|z|=1}|P(z)|+\frac{\left(K^{n}-1\right)}{\left(K^{n}+1\right)} \operatorname{Min}_{|z|=K}|P(z)|+\frac{4 K^{n}}{\left(K^{n}+1\right)} \frac{\left|a_{n-1}\right|}{(n+1)} \mid \frac{\left(K^{n}-1\right)}{n}-(K-1)\right] \\
& \left.+\frac{4 K^{n}}{\left(K^{n}+1\right)}\left|a_{n-2}\right|\left[\left\{\frac{\left(K^{n}-1\right)-n(K-1)}{n(n-1)}\right\}-\left\{\frac{\left(K^{n-2}-1\right)-(n-2)(K-1)}{(n-2)(n-3)}\right\}\right]+\operatorname{Min}_{|z|=K}|P(z)|\right\}, \text { if } n>3 .
\end{aligned}
$$

Equivalently

$$
\begin{aligned}
\operatorname{Max}_{|z|=K}\left|D_{\alpha} P(z)\right| \geq & \frac{n(|\alpha|-K) K^{n-1}}{\left(K^{n}+1\right)}\left\{\operatorname{Max}_{|z|=1}|P(z)|+\operatorname{Min}_{|z|=K}|P(z)|+\frac{2\left|a_{n-1}\right|}{(n+1)}\left[\frac{\left(K^{n}-1\right)}{n}-(K-1)\right]\right. \\
& \left.+2\left|a_{n-2}\right|\left[\left\{\frac{\left(K^{n}-1\right)-n(K-1)}{n(n-1)}\right\}-\left\{\frac{\left(K^{n-2}-1\right)-(n-2)(K-1)}{(n-2)(n-3)}\right\}\right]\right\}, \text { if } n>3 .
\end{aligned}
$$

Since $D_{\alpha} P(z)$ is a polynomial of degree $n-1$ and $K \geq 1$, therefore by using Lemma 1 , we get

$$
\operatorname{Max}_{|z|=K}\left|D_{\alpha} P(z)\right| \leq K^{n-1} \operatorname{Max}_{|z|=1}\left|D_{\alpha} P(z)\right|
$$

Combining (16) and (15) we have

$$
\begin{aligned}
\operatorname{Max}_{|z|=1}\left|D_{\alpha} P(z)\right| \geq & \frac{n(|\alpha|-K)}{\left(K^{n}+1\right)}\left\{\operatorname{Max}_{|z|=1}|P(z)|+\operatorname{Min}_{|z|=K}|P(z)|+\frac{2\left|a_{n-1}\right|}{(n+1)}\left[\frac{\left(K^{n}-1\right)}{n}-(K-1)\right]\right. \\
& \left.+2\left|a_{n-2}\right|\left[\left\{\frac{\left(K^{n}-1\right)-n(K-1)}{n(n-1)}\right\}-\left\{\frac{\left(K^{n-2}-1\right)-(n-2)(K-1)}{(n-2)(n-3)}\right\}\right]\right\}, \text { if } n>3 .
\end{aligned}
$$

This completes the proof of Theorem 2 .

\section{Acknowledgements}

The authors are extremely grateful to the referee for his valuable suggestions.

\section{References}

[1] A. Aziz and Q. M. Dawood, "Inequalities for a Polynomial and its Derivative," Journal of Approximation Theory, Vol. 54, No. 3, 1998, pp. 306-313.

[2] A. Aziz and N. A. Rather, "A Refinement of a Theorem of Paul Turán Concerning Polynomials,” Journal of Mathematical Inequality Application, Vol. 1, No. 2, 1998, pp. 231-238.

[3] N. G. de Bruijn, "Inequalities Concerning Polynomials in the Complex Domain,” Nederl. Akad. Wetench. Proc. Ser. A, Vol. 50, 1947, pp. 1265-1272; Indagationes Mathematicae, Vol. 9, 1947, pp. 591-598.

[4] K. K. Dewan, N. Singh and A. Mir, "Growth of Polynomials not Vanishing inside a Circle,” International Journal of Mathematical Analysis, Vol. 1, No. 11, 2007, pp. 529-538.

[5] N. K. Govil, “On the Derivative of a Polynomial,” Proceedings of the American Mathematical Society, Vol. 41, 1973, pp. 543-546. 
doi:10.1090/S0002-9939-1973-0325932-8

[6] P. D. Lax, "Proof of a Conjecture of P. Erdös on the Derivative of a Polynomial," American Mathematical Society, Vol. 50, No. 8, 1994, pp. 509-513.

[7] M. A. Malik, "On the Derivative of a Polynomial,” Journal of the London Mathematical Society, Vol. 2, No. 1, 1969, pp. 57-60. doi:10.1112/jlms/s2-1.1.57
[8] Polya and G. Szegö, “Ausgaben und Lehratze ous der Analysis,” Springer-Verlag, Berlin, 1995.

[9] W. M. Shah, "A Generalization of a Theorem of Paul Turán,” Journal Ramanujan Mathematical Society, Vol. 11, 1996, pp. 67-72.

[10] P. Turán, “Über die Ableitung von Polynomen,” Compositio Mathematica, Vol. 7, 1939, pp. 89-95. 